\title{
Ground-state entanglement of spin-1 bosons undergoing superexchange interactions in optical superlattices
}

\author{
Artur Barasiński, ${ }^{1}$ Wiesław Leoński, ${ }^{1}$ and Tomasz Sowiński ${ }^{2,3}$ \\ ${ }^{1}$ Quantum Optics and Engineering Division, Institute of Physics, \\ University of Zielona Góra, Z. Szafrana 4a, 65-516 Zielona Góra, Poland \\ ${ }^{2}$ Institute of Physics of the Polish Academy of Sciences, Al. Lotników 32/46, 02-668 Warsaw, Poland \\ ${ }^{3}$ Center for Theoretical Physics of the Polish Academy of Sciences, Al. Lotników 32/46, 02-668 Warsaw, Poland
}

\begin{abstract}
We discuss a model with ultra-cold atoms confined in optical superlattices. In particular, we study the ground-state properties of two spin-1 bosons trapped in a double-well potential. Depending on the external magnetic field and biquadratic interactions different phases of magnetic order are realized. Applying von Neumann entropy and number of relevant orbitals, we quantify the bipartite entanglement between particles. Changing the values of the parameters determining superlattices, we can switch the system between differently entangled states.
\end{abstract}

\section{INTRODUCTION}

Quantum entanglement is one of fundamental concepts in physics and it plays a crucial role in quantum information theory [1]. Without idea of entanglement, it is not possible to understand many spectacular properties of quantum systems or phenomena related to them. On can mention at this point teleportation $[2,6]$, quantum cryptography [7, 8], topological order [9, 10] or quantumness of physical systems [1] etc. In a context of strongly correlated many-body systems, together with quantum statistics, the entanglement between indistinguishable particles is responsible for nonintuitive properties of macroscopic systems [12]. The relation between the ground-state entanglement and the quantum phase transitions provide a bridge between quantum information theory and condensed matter physics [13 15]. For instance, transitions from the product states to strongly entangled ones in a two-qubit Heisenberg nuclear spin chain have been experimentally observed in systems affected by varying external magnetic field [16, 17].

In recent years, a wide perspective for experimental realization of the spin chains systems have been found in models involving ultra-cold atoms trapped in optical lattices [18, 19], ultracold quantum gates 20], or coupled quantum dots 21] etc. Contrary to the solidstate physics, ultra-cold atomic systems can be quite well tuned to situations in which their properties are well described by simplified models of condensed matter theory. Moreover, they permit to engineer and manipulate quantum states in single lattice sites as well as collectively on a whole lattice. It is possible to tune all relevant parameters almost adiabatically, even through quantum phase transitions [22, 23] and in consequence, to test scaling invariance and universality [24]. Such systems have also opened completely new possibilities for answering to fundamental questions of quantum information theory related to transitions from the product to entangled states, quantum state transfers, quantum magnetism, spin dynamics, etc. [25 29]. In this way, ultra-cold atomic systems become dedicated quantum simulators [30, 31] for condensed matter problems, where fundamental param- eters of theoretical models can be controlled experimentally.

Properties of interacting ultra-cold atoms confined in optical lattices are typically described in the language of Hubbard-like models. In the simplest case of spinless bosons interacting repulsively via short-range delta-like interactions, the Bose-Hubbard model has only two competing terms related to the single-particle tunneling to the neighboring site and to the additional energy cost when two bosons meet in given site 32. Extended Hubbard models (for fermions and bosons) originate in taking into account higher bands of optical lattices, longrange interactions, and internal structure of interacting particles 33 37]. In particular, scenarios when tunneling is dominated by interactions, all Hubbard models can be perturbatively simplified and can be rewritten to appropriate lattice-spin models. For example, when the standard Bose-Hubbard model is considered and the system remains in the Mott-insulator phase, one can find the most relevant corrections by treating the tunneling as a virtual process in the second order of perturbation theory [38]. With this observation, Simon et al. mimicked experimentally the one-dimensional Ising model 39]. In more general case, when internal structure of ultra-cold atoms can not be neglected, the low energy effective Hamiltonian requires superexchange interactions [40]. Then, the corresponding spin model also includes higher powers of the scalar products of spin operators at neighboring sites, $H=\sum_{k} J_{k}\left(\hat{\boldsymbol{S}}_{i} \cdot \hat{\boldsymbol{S}}_{j}\right)^{k}$ [41 45]. For example, for the case of spin-1 bosons the system is described by the Heisenberg model with additional biquadratic term, and is called Quadratic-Biquadratic Heisenberg (QBH) model [41, 42, 46]. So far, mostly the linear Heisenberg spin-models were intensively studied over wide range of parameters [47 52. The QBH model have attracted great interest mainly due to a wealth of possible quantum phases, predicted in its antiferromagnetic case, and also in i.e. Haldane, nematic, dimerized, trimerized phases etc. [53 55], as well. The QBH model was also successfully applied in the studies concerning magnetic properties and energy levels distribution in several real materials [56 60], where the validity of the bi- 
quadratic interaction has been emphasized.

Motivated by all these observations, we shall discuss here the properties of spin- 1 bosons (for example alkali atoms of 23 -sodium or 87-rubidium) confined in optical superlattice, i.e. the lattice created by interference of two independent laser standing waves with commensurate frequencies ratio equal to 2 . Such lattice is characterized by a structure involving weakly coupled doublewell potentials and therefore, it could be a quite good arena for studying two-site Hubbard models. Moreover, in the limit of strong repulsions this model can be simplified to the two-site QBH model [41, 42, 46]. We should note that in [61] the authors have concentrated on the effect of the asymmetry of the double-well potential in the realization of various types of magnetic order. They also discussed some aspects related to the bipartite entanglement between sites in a quite limited regime of parameters. Here, we complement and extend these studies assuming that full range of parameters of resulting Hamiltonian can be reached experimentally. It can be done directly (for instance by using the optical Feshbach resonances [53, 62]) or by performing some kind of "quantum simulation" where the system was prepared in its excited state (like it was proposed [46, 54]). In particular, our main goal is to analyze the influence of the biquadratic interaction to the ground-state entanglement.

The paper is organized as follows. In Sec. II we introduce the model studied here and present its theoretical background. In Sec. III we give a full analysis of the spectrum of two-site Hamiltonian, and in Sec. IV we present phase diagram of the ground-state in a whole accessible range of parameters. Sec. V is devoted to the presentation of main results related to the entanglement generation in the ground state. Finally, we present our conclusions in Sec. V.

\section{TWO-SITE SPIN-MODEL HAMILTONIAN}

In this article, we consider spin-1 ultra-cold bosons confined in a one-dimensional superlattice potential. We assume that dynamics is frozen in two perpendicular directions and the particles remain in the ground state of confining potential. Moreover, that laser beams are configured in such a way that one can treat system as independent double-well potentials. In other words, we assume that tunneling amplitudes between potential dimmers are much smaller than the tunnelings inside the dimer. Such situation is well described with two-side Bose-Hubbard model of the form

$$
\begin{aligned}
H & =-t\left(\hat{a}_{L \sigma}^{\dagger} \hat{a}_{R \sigma}+\hat{a}_{R \sigma}^{\dagger} \hat{a}_{L \sigma}\right)+\frac{U_{0}}{2} \sum_{i=L, R} \hat{n}_{i}\left(\hat{n}_{i}-1\right)+ \\
& +\frac{U_{2}}{2} \sum_{i=L, R}\left(\hat{\boldsymbol{S}}_{i}^{2}-2 \hat{n}_{i}\right)-\gamma \boldsymbol{B} \cdot\left(\hat{\boldsymbol{S}}_{L}+\hat{\boldsymbol{S}}_{R}\right)
\end{aligned}
$$

where $\hat{a}_{i \sigma}$ annihilates boson in spin state $\sigma \in\{-1,0,1\}$ at left $(L)$ or right $(R)$ well, $\hat{n}_{i}=\sum_{\sigma} \hat{a}_{i \sigma}^{\dagger} \hat{a}_{i \sigma}$ is a to- tal number of particles at give site, whereas $\hat{\boldsymbol{S}}_{L}$ and $\hat{\boldsymbol{S}}_{R}$ are total spin operators. The parameters $U_{0}$ and $U_{2}$ describe repulsive interaction between two particles confined in one lattice site. They are proportional to the s-wave scattering lengths $a_{0}$ and $a_{2}$ when total spin of colliding particles is $S=0$ and $S=2$, respectively (see for example [42]). The last term in the Hamiltonian (11) describes linear Zeeman effect in the external magnetic field $\boldsymbol{B}$. The parameter $\gamma=g \mu_{B}$, where $\mu_{B}$ is the Bohr magneton and $g$ is the Landé factor. In further analysis we will assume that external magnetic field is oriented along $z$ axis. It is worth to notice that inversion of the spin-axis quantization is equivalent to the inversion of the magnetic field. Therefore, without loosing generality, one can assume that magnetic field $B^{z}$ is not negative. All predictions for $B^{z}<0$ have their counterparts with opposite sign of the spins in $B^{z}>0$. In fact, the Hamiltonian (11) is an effective Hamiltonian, general enough to be applied in description of a large family of physical systems. For instance, it was used in discussion of various interesting phenomena appearing in Kerrlike quantum-optical or nano- systems models, such as photon (phonon) blockade 63 65] (sometimes called as nonlinear quantum scissors [66 69]) or quantum-chaotic behavior 70 74].

Our aim is to study the system with unit filling in the strongly repulsive regime, i.e. when non-local tunneling term in the Hamiltonian (11) can be treated as a small perturbation (when compared with sum of local interaction terms). Therefore, we rewrite the Hamiltonian as the effective one in the second order of perturbation in $t$ [41, 42]:

$$
H=J_{0}+J_{1} \hat{\boldsymbol{S}}_{L} \cdot \hat{\boldsymbol{S}}_{R}+J_{2}\left(\hat{\boldsymbol{S}}_{L} \cdot \hat{\boldsymbol{S}}_{R}\right)^{2}-\gamma B^{z}\left(\hat{S}_{L}^{z}+\hat{S}_{R}^{z}\right),
$$

where $J_{1}=-\frac{2 t^{2}}{U_{2}}, J_{2}=-\frac{2 t^{2}}{3 U_{2}}-\frac{4 t^{2}}{3 U_{0}}$ and $J_{0}=J_{1}-J_{2}$.

Since we shall study effects of the biquadratic term in the Hamiltonian (2), it is convenient to parametrize the Hamiltonian (2) with three dimensionless parameters

$$
H=\lambda \hat{\boldsymbol{S}}_{L} \cdot \hat{\boldsymbol{S}}_{R}+\tan \theta\left(\hat{\boldsymbol{S}}_{L} \cdot \hat{\boldsymbol{S}}_{R}\right)^{2}-h\left(\hat{S}_{L}^{z}+\hat{S}_{R}^{z}\right),
$$

where $\lambda=J_{1} /\left|J_{1}\right|= \pm 1$ is a sign of a linear term, $\tan \theta=J_{2} /\left|J_{1}\right|$, and $h=\gamma B^{z} /\left|J_{1}\right|$. For convenience, we also set the energy scale in such a way that $J_{0}=0$. Positive (negative) $\lambda$ favors antiferromagnetic (ferromagnetic) orientation of spins. It should be emphasized that although, from the model point of view, the angle $\theta$ lies within the interval $(-\pi / 2, \pi / 2)$, for the system of ultracold atoms when the ratio is typically $U_{2} / U_{0}>0$, angle $\theta$ can not cover this range completely [53]. Nevertheless, the experimental scheme where a whole range of $\theta$ can be obtained was also proposed [46, 54]. Moreover, in contrast to usual condensed matter systems (see for example [40]), ultra cold atoms make it possible to engineer experimental setups where biquadartic coupling is larger than linear one. 

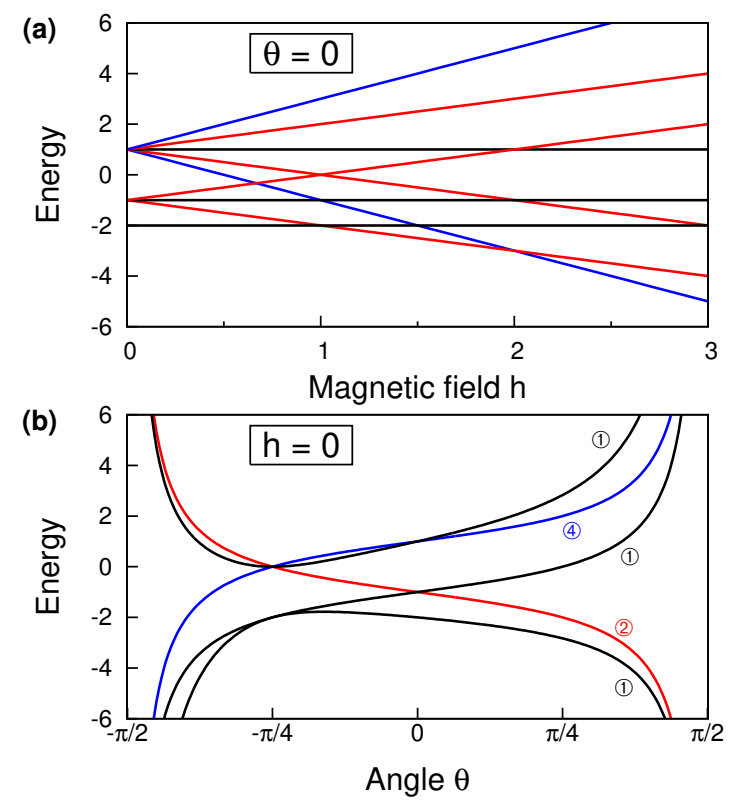

FIG. 1. Spectrum of the Hamiltonian (3) for $\lambda=1$ in two limiting situations: (a) vanishing biquadratic interaction $\theta=$ 0 , (b) vanishing external magnetic field $h=0$. In (a) different colors corresponds to states with different total spin $S^{z}\left(S^{z}=\right.$ 0 black line, $S^{z}= \pm 1$ red line, $S^{z}= \pm 2$ blue line); in (b) circled numbers denote degree of the degeneracy of the states.

It should be also underlined that the effective Hamiltonian (31) can be derived from the more general Hubbardlike Hamiltonian (11) only for repulsive interactions. Only for this case, the Mott-Insulator phase with one particle in each lattice site is a true ground-state of the system, in the limit of vanishing tunneling. This means that only negative sign of $\lambda$ can be obtained in this framework. However, it was shown recently that positive $\lambda$ can be effectively engineered by preparing system in its excited state [46, 54]. In consequence, although such system is not in the true ground-state, it can be quite well described with use of the effective Hamiltonian (3) with positive $\lambda$ and for long time-scales.

\section{SPECTRUM OF THE EFFECTIVE HAMILTONIAN}

The Hamiltonian (3) commutes with the total spin operator $\hat{S}^{z}=\hat{S}_{L}^{z}+\hat{S}_{R}^{z}$. Therefore, it can be diagonalized in the subspaces of given spin. All these subspaces are spanned together by nine natural eigenvectors of total spin $\left|\sigma, \sigma^{\prime}\right\rangle=\hat{a}_{L \sigma}^{\dagger} \hat{a}_{R \sigma^{\prime}}^{\dagger}|\mathrm{vac}\rangle$. Performing exact diagonalization of the Hamiltonian, one can find a local groundstate for each subspace, corresponding to a given spin. In a consequence, the true ground-state of the system is the one with the lowest energy. It is quite obvious that the states $|1,1\rangle$ and $|-1,-1\rangle$ with the largest absolute total spin $\left(S^{z}= \pm 2\right)$ are the eigenstates of the Hamiltonian (3) for any set of dimensionless parameters. It comes directly from the fact that they are only states with the largest (smallest) total spin. Their eigenenergies are equal to $E_{ \pm 2}=\lambda+\tan \theta \mp 2 h$, respectively. For these states we observe no entanglement between two sites.

The subspaces with total spin $S^{z}= \pm 1$ are spanned by two natural eigenvectors: $|1,0\rangle$ and $|0,1\rangle$ for $S^{z}=1$, and $|-1,0\rangle$ and $|0,-1\rangle$ for $S^{z}=-1$. Due to the presence of additional $Z_{2}$ symmetry of the Hamiltonian (3), namely the mirror left-right symmetry, the eigenstates of the Hamiltonian in these subspaces are also easy to find and they are represented by two maximally entangled states (MES) - Bell states. For $S^{z}=1$ these states are

$$
\| 1 ; \pm\rangle=\frac{|0,1\rangle \pm|1,0\rangle}{\sqrt{2}}
$$

with corresponding eigenenergies $E_{1 ; \pm}= \pm(\lambda+\tan \theta)-h$. Similarly, for $S^{z}=-1$ these states are

$$
\|-1 ; \pm\rangle=\frac{|0,-1\rangle \pm|-1,0\rangle}{\sqrt{2}}
$$

and corresponding eigenenergies $E_{-1 ; \pm}= \pm(\lambda+\tan \theta)+$ $h$. The most interesting situation is in the remaining subspace of total spin $S^{z}=0$. This subspace is spanned by three natural eigenvectors $|-1,1\rangle,|0,0\rangle$, and $|1,-1\rangle$. Due to the mirror symmetry of the Hamiltonian, mentioned above three eigenstates of the Hamiltonian in this subspace have following forms:

$$
\begin{aligned}
\| 0 ; 0\rangle\rangle & =\frac{|-1,1\rangle-|1,-1\rangle}{\sqrt{2}}, \\
\| 0 ; \pm\rangle\rangle & =\cos \alpha_{ \pm} \frac{|-1,1\rangle+|1,-1\rangle}{\sqrt{2}} \pm \sin \alpha_{ \pm}|0,0\rangle,
\end{aligned}
$$

with corresponding eigenenergies:

$$
\begin{aligned}
E_{0 ; 0} & =-\lambda+\tan \theta, \\
E_{0 ; \pm} & =(-\lambda+\tan \theta \pm \delta) / 2,
\end{aligned}
$$

where $\delta=\left[9(\lambda+\tan \theta)^{2}-4 \lambda \tan \theta\right]^{1 / 2}$. The mixing angles $\alpha_{ \pm}$are related to the parameters of the Hamiltonian by the condition $\cos \alpha_{ \pm}=\sqrt{\left|E_{0 ; \pm}\right| / \delta}$. It can be easily checked that the state $\| 0 ;-\rangle\rangle$ is a ground state in considered subspace for any set of parameters of the Hamiltonian. In addition, for particular set of parameters $\lambda=1$ and $\theta_{0}=-\pi / 4$, it is degenerated with the state $\| 0 ; 0\rangle\rangle$. As it will be explained in Sec. V, at this point the state $\| 0 ;-\rangle\rangle$ changes its character from threedimensional triplet (for $\theta>\theta_{0}$ ) to three-dimensional singlet (for $\theta<\theta_{0}$ ) Bell state.

The whole spectrum of the Hamiltonian for two cases: vanishing biquadratic interaction $(\theta=0)$ and vanishing external magnetic field $(h=0)$, when positive linear interaction is assumed $(\lambda=1)$, are plotted in Fig. 1. As we see there, the presence of biquadratic term does not lift the degeneracy completely (the remaining multiplicity of energy levels is marked by numbers in circles). 

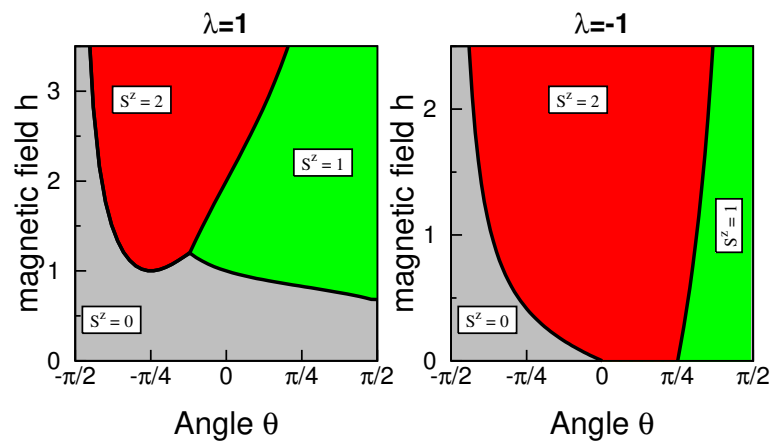

FIG. 2. Ground-state phase diagram of the system. For given angle $\theta$ and magnetic field $h$ ground-state of the system belongs to the one of three subspaces characterized with total spin $S^{z}$. Note that properties of the ground-state highly depend on the sign of the linear coupling term $\lambda$. The phase diagram for negative magnetic field $h$ has identical phases but with opposite total spin.

\section{GROUND-STATE PHASE DIAGRAM}

To get more clear picture of possible scenarios we present in Fig. 2 the ground-state phase diagram of the system. This diagram is obtained with application of exact diagonalization of the Hamiltonian (3). We see that for given values of the parameters describing the Hamiltonian and magnetic filed $h>0$, the true ground-state of the system belongs to the one of three subspaces of total spin $S^{z}=0,1,2$. Obviously, for negative magnetic field $h<0$ the phase diagram is exactly the same but quantum phases correspond to opposite sign of the total spin.

As it is seen from Fig 2 the phase diagram crucially depends on the sign of the linear coupling $\lambda$. It is worth to notice that for the both cases $\lambda= \pm 1$, the ferrimagnetic-like quantum phase with total spin $S^{z}=1$ is the only phase which is confined by some minimal values of the biquadratic interaction. However, one should keep in mind that the ground state is always in the ferromagnetic phase (with the largest total spin $S^{z}=2$ ) for large enough values of magnetic field, whereas the most interesting antiferromagnetic-like phase with total spin $S^{z}=0$, can be reached only for sufficiently small values of magnetic field. When additionally, $\lambda$ is positive, this phase $\left(S_{z}=0\right)$ can be reached for any value of biquadratic interactions. However, when $\lambda$ is negative one needs also negative biquadratic interaction to reach antiferromagnetic-like phase.

Due to the fact that in the Hamiltonian (3) there is no term corresponding to the coupling states with different $S^{z}$, the phases are separated by sharp borders. All quantum phase transitions appearing in the system are of the first order - they are characterized by discontinuities in the first derivative of the ground- state energy. In other words, at the transition points the ground-state of the system ,,jumps" from one subspace of total spin to the other one. For example, when $\lambda=1$ and $\theta=0$, we see that system undergoes two transitions for $h=1$ and for $h=2$. These transitions are directly related to the crossings of the energy levels shown in Fig,1b, and at those points magnetization of the ground-state changes its value suddenly.

\section{QUANTUM CORRELATIONS IN THE GROUND STATE}

In this section we will discuss an entanglement properties of the ground state more deeply. To study them we shall use two quantities to determine the entanglement present in the system. One of them is the following correlation parameter, introduced in [75]:

$$
\mathcal{K}\left(\hat{\rho}_{L}\right)=\left(\sum_{i} \eta_{i}^{2}\right)^{-1}
$$

where $\eta_{i}$ are the eigenvalues of the left-site reduced density matrix $\hat{\rho}_{L}=\operatorname{Tr}_{R}|G\rangle\langle G|$ obtained from the full, twosite ground state density matrix $|G\rangle\langle G|$ by tracing out degrees of freedom of the right-well (obviously this parameter can be derived for the right-site without changing the results). The parameter $\mathcal{K}$ gives an effective number of single-particle orbitals occupied in the given many-body state. In particular, when the one-site density matrix has $n$ equal eigenvalues, then $\mathcal{K}=n$. Additionally, when we are dealing with product states $\mathcal{K}=1$. This parameter $(\mathcal{K})$ can be very useful is studies of bipartite systems [76].

Other measure of the system entanglement, that we shall apply in this paper is von Neumann entropy. This measure is commonly used in numerous papers, and can be defined as

$$
\mathcal{S}\left(\hat{\rho}_{L}\right)=\operatorname{Tr}\left(\hat{\rho}_{L} \log _{2} \hat{\rho}_{L}\right)=\sum_{i} \eta_{i} \log _{2} \eta_{i}
$$

This entropy is even more interesting than the number of relevant orbitals $\mathcal{K}$ since it is directly related to the properties of the system in the thermodynamic limit, as well as in quantum information context. Entropy defined in (8) for bipartite system ranges from 0 for completely disentangled (product) states to $\log _{2} D$ for MES defined in $D$-dimensional Hilbert space. On the other hand, we can discuss our system in terms of quantum information theory. For such a case, we treat the system as that of two $D$-dimensional qudits [77]. Therefore, besides the total spin previously used, the phases can be explicitly distinguished with respect to the quantum state of the system and consequently, to the dimension of the Hilbert subspaces in which these states are defined. Therefore, the system found in a given phase can be represented by the corresponding to it quantum information theory objects. For instance, the bipartite system in the antiferromagnetic-like phase is described by the state defined in three-dimensional Hilbert subspace and hence, 
can be treated as qutrit-qutrit system. Similarly, the system in ferrimagnetic-like phase can be considered as a qubit-qubit one.

The quantities $\mathcal{K}$ and $\mathcal{S}$ characterize global properties of shared entanglement and they can be insensitive to the internal structure of the state. For example, there are various MES which are characterized with the same $\mathcal{K}$ and $\mathcal{S}$. Since we are interested in two, particular generalized Bell states [78]:

$$
\begin{aligned}
& \left|3, \mathrm{~B}_{\mathrm{S}}\right\rangle=\frac{1}{\sqrt{3}}(|1,-1\rangle+|-1,1\rangle-|0,0\rangle), \\
& \left|3, \mathrm{~B}_{\mathrm{T}}\right\rangle=\frac{1}{\sqrt{3}}(|1,-1\rangle+|-1,1\rangle+|0,0\rangle),
\end{aligned}
$$

to overcome this problem we shall measure the relative distance between the ground state of the system $|\mathrm{G}\rangle$ and these Bell states. Such relative distances are represented by the fidelities $\mathcal{F}_{S}=\left|\left\langle 3, \mathrm{~B}_{\mathrm{S}} \mid \mathrm{G}\right\rangle\right|^{2}$ and $\mathcal{F}_{T}=\left|\left\langle 3, \mathrm{~B}_{\mathrm{T}} \mid \mathrm{G}\right\rangle\right|^{2}$, respectively.

Note that, by definition, these states are not orthogonal. In consequence, even if the fidelity calculated in respect to one of them is equal to one, the remaining one is not zero. (see Fig. 3k). Thus, only simultaneous inspection of all three parameters $\left(\mathcal{S}, \mathcal{K}\right.$ and $\left.\mathcal{F}_{T, S}\right)$ can give insight into discussed physical situation.

\section{A. Positive linear coupling $(\lambda=+1)$}

We shall discuss various cases interesting from the point of view of the entanglement generation processes. One of the possible situations (the most trivial one) is that of the total spin $S^{z}= \pm 2$. Simply, being in these phases the system does not exhibit any nonlocal correlations. for this case $\mathcal{K}$ and $\mathcal{S}$ are equal to 1 and 0 respectively, and therefore, the ground-state is a product state.

Whenever the ground-state of the system is in the ferrimagnetic-like phase (total spin $S^{z}= \pm 1$ ) the system remains in singlet Bell state for qubit-qubit system, $\| 1,-\rangle$, with $\mathcal{K}=2$ and entropy $\mathcal{S}=1$. It is worth mentioning that in this phase the state $\| 1,-\rangle\rangle$ is exact ground-state for any values of the parameters appearing in the Hamiltonian. Therefore, the entanglement of the ground-state in this phase is robust to any changes of these parameters.

The most interesting situation is in the antiferromagnetic-like phase with $S^{z}=0$. For this case the ground-state of the system, and in consequence the degree of system entanglement, crucially depend on the mixing angle $\theta$. In Fig 3 a entropy $\mathcal{S}$ and the number of relevant orbitals $\mathcal{K}$ for this phase are plotted. They can be calculated analytically directly from the
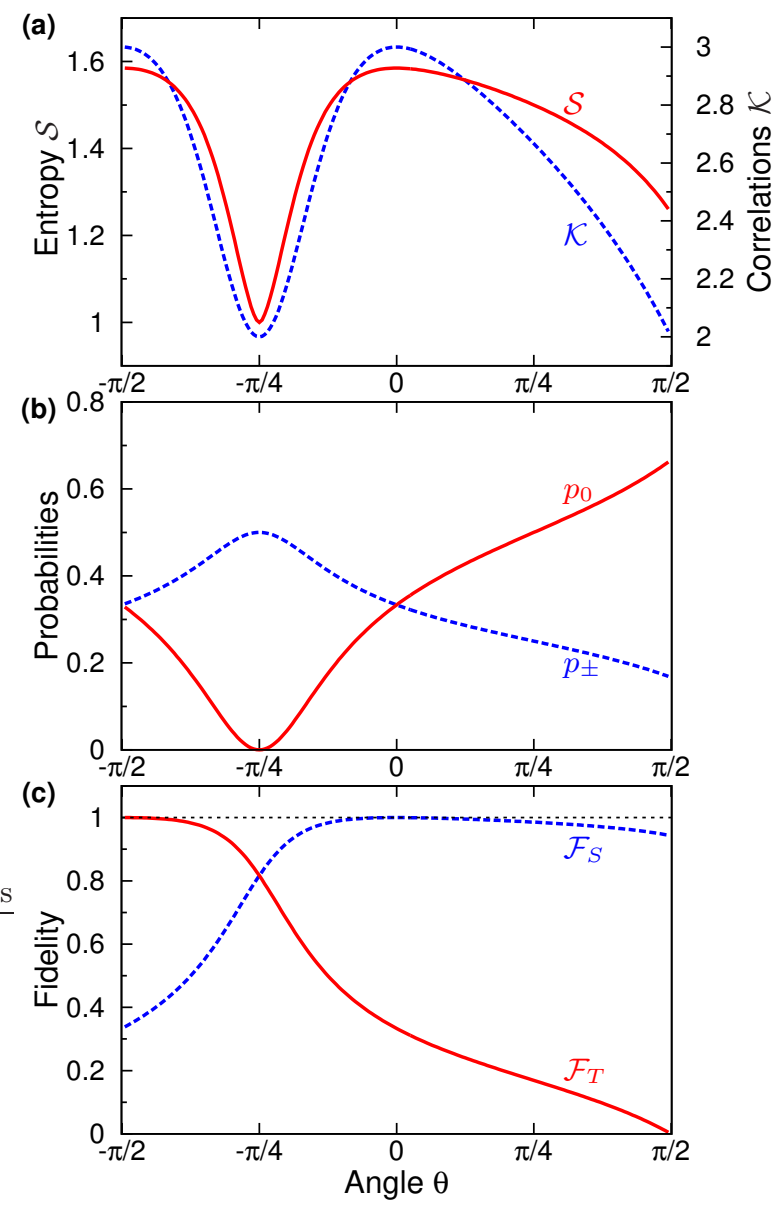

FIG. 3. Ground-state properties for positive linear coupling $\lambda$ in the phase with total spin $S^{z}=0$ as a function of the mixing angle $\theta$. (a) Number of relevant orbitals $\mathcal{K}$ (blue dashed line) and the von Neumann entropy $\mathcal{S}$ (red solid line), (b) probabilities $p_{0}$ and $p_{ \pm}$of finding the ground-state in appropriate two-site product states (as explained in the main text), and (c) fidelities $\mathcal{F}_{S}\left(\mathcal{F}_{T}\right)$ between the ground-state of the system and singlet (triplet) qutrit-qutrit Bell state.

equation (5b)

$$
\begin{aligned}
\mathcal{S}= & \frac{E_{0 ;-}}{\delta} \log _{2}\left(\frac{\left|E_{0 ;-}\right|}{2 \delta}\right)+ \\
& \frac{2(\lambda+\tan \theta)^{2}}{\delta E_{0 ;-}} \log _{2}\left(\frac{2(\lambda+\tan \theta)^{2}}{\delta\left|E_{0 ;-}\right|}\right), \\
\mathcal{K}= & \frac{2 \delta^{2} E_{0 ;-}^{2}}{8(\lambda+\tan \theta)^{4}+E_{0 ;-}^{4}}
\end{aligned}
$$

The both quantities varies with the biquadratic interaction strength and hence, different qutrit-qutrit MES can be generated (Fig $3 \mathrm{a}$ ). In Figs $3 \mathrm{~b}$ and $3 \mathrm{k}$ other quantities which allow to fully characterize properties of the ground-state $|G\rangle$ are shown. In particular, in Fig $3 b$ we plot probabilities of finding the ground-state in two site product states, i.e. $p_{ \pm}=|\langle\mp 1, \pm 1 \mid \mathrm{G}\rangle|^{2}$ (blue dashed line) and $p_{0}=|\langle 0,0 \mid \mathrm{G}\rangle|^{2}$ (red solid line). Finally, in Fig 3 ; we 
present the fidelities $\mathcal{F}_{S}$ and $\mathcal{F}_{T}$.

As it is seen from Fig 3 , deeply in the repulsion biquadratic interactions regime $(\theta \rightarrow-\pi / 2)$, the entropy $\mathcal{S}=\log _{2} 3$, whereas the number of relevant orbitals $\mathcal{K}=3$. For such a case the ground state of the system is the maximally entangled triplet state

$$
|G\rangle \stackrel{\theta \rightarrow-\pi / 2}{\longrightarrow}\left|3, B_{\mathrm{T}}\right\rangle \text {. }
$$

When biquadratic interaction grows, the both: $\mathcal{S}$ and $\mathcal{K}$ start to decrease rapidly. Thus, for $\theta_{0}=-\pi / 4$ the entropy $\mathcal{S}=\log _{2} 2$ and $\mathcal{K}=2$, what is related to vanishing of the probability $p_{0}$. In consequence, at this point the ground-state, initially specified in $3 \otimes 3$-dimensional Hilbert space, reduces to the state defined in $2 \otimes 2$ subspace. This state can be written as

$$
\left|2, \mathrm{~B}_{\mathrm{T}}\right\rangle=\frac{1}{\sqrt{2}}(|1,-1\rangle+|-1,1\rangle) .
$$

In fact, it is Bell MES defined in $2 \otimes 2$ Hilbert space, and we deal here with a qubit-qubit system. Moreover, one should remember that for $\theta=\theta_{0}$ the ground-state $\left|2, \mathrm{~B}_{\mathrm{T}}\right\rangle$ is degenerate with other Bell state $\| 0,0\rangle\rangle$.

When $\theta>\theta_{0}$ the probability amplitude $\langle 0,0 \mid \mathrm{G}\rangle$ changes its sign and in consequence, the ground-state changes its nature, switching form triplet-like to singletlike state (they are not perfect triplet and singlet states, as some amount of the probability corresponding to other states is present in the system). Such switching can be realized in practical realizations for instance, by adiabatic changes of the parameters of the Hamiltonian. Moreover, from Fig 3 , we see that the fidelity $\mathcal{F}_{S}$ increases and becomes larger than $\mathcal{F}_{T}$ for $\theta>\theta_{0}$ and then, reaches its maximal value $\left(\mathcal{F}_{S}=1\right)$ for vanishing biquadratic interaction $(\theta=0)$. At this point the singlet Bell state is generated

$$
|\mathrm{G}\rangle \stackrel{\theta=0}{=}\left|3, \mathrm{~B}_{\mathrm{S}}\right\rangle,
$$

what is manifested by $\mathcal{S}=\log _{2} 3$ and $\mathcal{K}=3$.

For attractive interactions $(\theta>0)$ the ground-state remains almost exactly in the singlet state $\left|3, \mathrm{~B}_{\mathrm{S}}\right\rangle$, i.e. the fidelity $\mathcal{F}_{S}$ decreases but not more than $\sim 10 \%$ from the unity. Moreover, we see that for this case the entropy decreases, the same as the number of relevant orbitals. In the limit $\theta \rightarrow \pi / 2$ we have $\mathcal{K}=2$. This fact shows that only two relevant orbitals are involved, and might suggest that we are dealing with the same situation as that for $\theta=\theta_{0}$. However, for this situation the entropy $\log _{2} 3>\mathcal{S}>\log _{2} 2$. In consequence, although the state of our system is defined in $3 \otimes 3$ Hilbert space, it is not MES. The fact that $\mathcal{K}=2$ is caused by the distribution of the probabilities $p_{0}$ and $p_{ \pm}$. As we can see from Fig $3 \mathrm{~b}$, for such situation the probability $p_{0}$ plays a dominant role and is four times higher then $p_{ \pm}$. Therefore, the sum of the both probability amplitudes, corresponding to $p_{ \pm}$, is equal to the amplitude corresponding to $p_{0}$. In consequence, the effective number of relevant orbitals is equal to 2 .
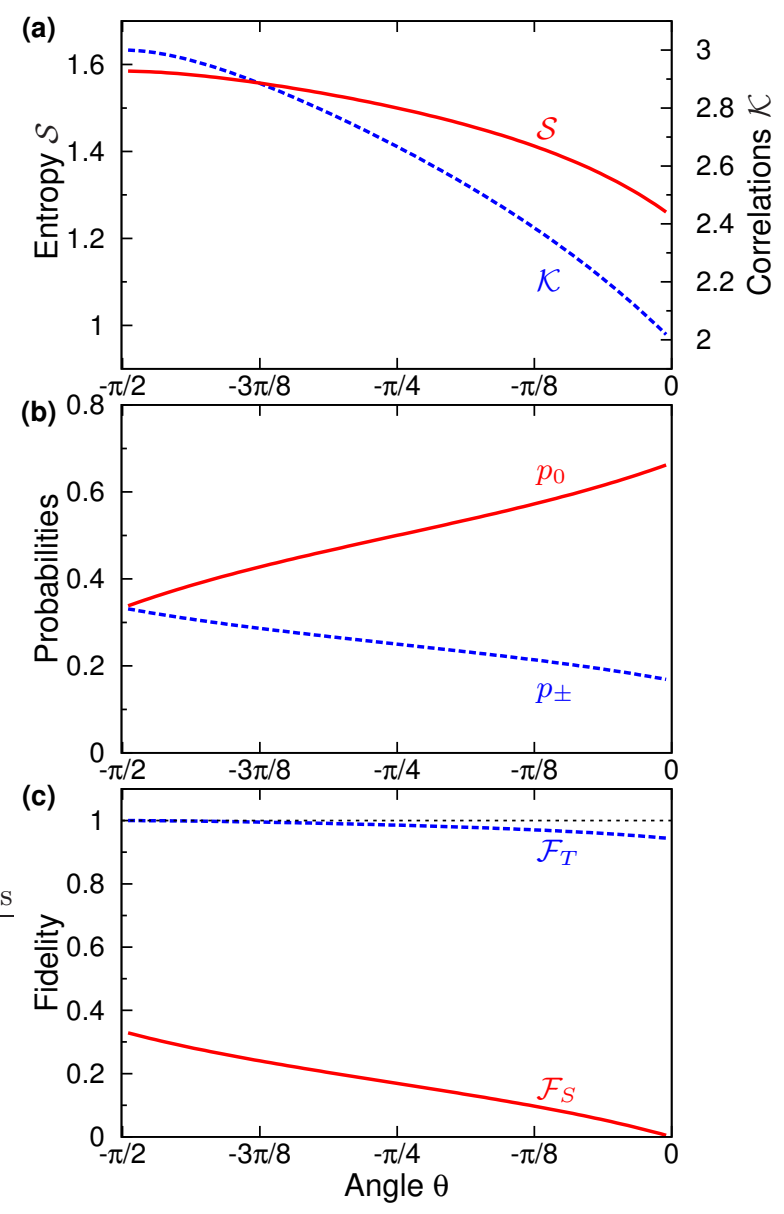

FIG. 4. Ground-state properties for negative linear coupling $\lambda$ in the phase with total spin $S^{z}=0$ as a function of the mixing angle $\theta$. (a) Number of relevant orbitals $\mathcal{K}$ (blue dashed line) and the von Neumann entropy $\mathcal{S}$ (red solid line), (b) Probabilities $p_{0}$ and $p_{ \pm}$of finding the ground-state in appropriate two-site product states (as explained in the main text), (c) Fidelities $\mathcal{F}_{S}$ and $\mathcal{F}_{T}$ between the ground-state of the system and singlet and triplet qutrit-qutrit Bell state respectively. In contrast to the case $\lambda=1$, the ground-state remains almost perfectly in the triplet qutrit-qutrit Bell state $\left|3, \mathrm{~B}_{\mathrm{T}}\right\rangle$ in a whole phase.

\section{B. Negative linear coupling $(\lambda=-1)$}

Similarly, correlations in the ground-state can be discussed for negative linear coupling $\lambda=-1$. As previously, for the phases characterized by total spin $S^{z}=2$ and $S^{z}=1$, the ground-state is product $|1,1\rangle$ and singlet qubit-qubit Bell state $\| 1,-\rangle\rangle$, respectively.

Situation changes for the case when total spin $S^{z}=0$, what can be observed only for negative values of the angle $\theta$. For such a situation all properties of the ground state can also be derived analytically. In particular, the formulas for the entropy $\mathcal{S}$ and the number of relevant orbitals $\mathcal{K}$ have the same form in eq. (10). They are plotted in Fig 4a. As it is seen from Fig 40, in contrast to the case $\lambda=1$, for negative linear coupling the ground- 
state of the system remains almost perfectly in the triplet qutrit-qutrit Bell state $\left|3, \mathrm{~B}_{\mathrm{T}}\right\rangle$. In the limit of infinite repulsive biquadratic interactions, the fidelity $\mathcal{F}_{T}$ is equal to 1 . Moreover, all three probabilities $p_{ \pm}$and $p_{0}$ are equal to $1 / 3$. All these means that for the cases when $\lambda=-1$, the degree of bipartite entanglement for the ground state is highly insensitive on the values of the parameters describing the superlattice. One should remember that such robustness appears when the system is in the phase with vanishing total spin $S^{z}=0$, and was not present for $\lambda=1$ (see the cases discussed in previous section).

\section{CONCLUSIONS}

We have discussed the model of two spin-1 bosons confined in the double-well potential of the optical superlattice and influenced by an external magnetic field. We showed that dependent on the values of the parameters appearing in the Hamiltonian, the ground-state can belong to different phases distinguished by total spin $S^{z}$, and the presence of non-linear biquadratic interaction considerably influences the system's properties. For experimentally accessible values of the parameters the ferro-, ferri- and antiferromagnetic-like phases have been identified and presented as a separated sectors in phasediagrams. We have pointed out that the sharp boundaries between sectors are related to the crossings of energies in the spectrum of the Hamiltonian. Subsequently, we have uniquely determined ground-state of the system of a given magnetization for each magnetic phase. In this paper we have concentrated on the possibility of generation of MES defined in $2 \otimes 2$ and $3 \otimes 3$ Hilbert spaces, and we have identified the set of parameters for which MES of different kinds can be achieved. What is important, applying changes in the parameters describing optical lattice, we can switch the system from one MES to another. For instance, we can continuously transform the state of our system from a singlet to a triplet Bell state. Moreover, it is possible to change the system's state from MES defined in $2 \otimes 2$ Hilbert subspace to that, defined in $3 \otimes 3$-dimensional space, and vice versa. Such switching performed within a single phase can be induced by adiabatic changes of the parameters of the Hamiltonian. For the cases when transitions are made between two phases, some additional interaction should be involved. Such interaction (for instance, interaction with external bath) is necessary, as the system has to change its total spin which commutes with the Hamiltonian.

In particular, we have found that in the ferrimagneticlike phase the ground-state is a qubit-qubit singlet Bell state regardless of the type of the Heisenberg interaction. Moreover, discussing the case of antiferromagneticlike phase, we have showed that the biquadratic term of Heisenberg interactions plays a crucial role in determining properties of the ground-state of the system. For $\lambda=-1$ the ground-state is the qutrit-qutrit triplet Bell state while for $\lambda=1$ the continuous transition from the qutrit-qutrit singlet to qutrit-qutrit triplet can be induced by adiabatic varying of biquadratic interaction. Moreover, at the transition point, the ground-state becomes the qubit-qubit triplet Bell state.

We believe that the system studied here can be a potential candidate for practical realization of a device which could be applied as a switchable tool for generation of various MES on demand.

\section{ACKNOWLEDGMENTS}

We would like to thank prof. A. Miranowicz for his valuable suggestions and discussions. The authors wish to thank the (Polish) National Science Center (grant No. DEC-2011/01/D/ST2/02019) for the support. Numerical calculations were performed in WCSS Wrocław (Poland).
[1] C. H. Bennett and D. P. DiVincenzo, "Quantum information and computation," Nature (London) 404, 247 (2000).

[2] D. Boschi, S. Branca, F. De Martini, L. Hardy, and S. Popescu, "Experimental realization of teleporting an unknown pure quantum state via dual classical and einstein-podolsky-rosen channels," Phys. Rev. Lett. 80, 1121 (1998).

[3] D. Bouwmeester, J. W. Pan, K. Mattle, M. Eible, H. Weinfurter, and A. Zeilinger, "Experimental quantum teleportation," Nature 390, 575 (1997).

[4] A. Miranowicz, "Optical-state truncation and teleportation of qudits by conditional eight-port interferometry," J. Opt. B: Quant. Semiclass. Opt. 7, 142 (2005).

[5] S. K. Ozdemir, K. Bartkiewicz, Y. X. Liu, and A. Miranowicz, "Teleportation of qubit states through dissipative channels: Conditions for surpassing the no-cloning limit," Phys. Rev. A 76, 042325 (2007).

[6] S. K. Goyal and T. Konrad, "Teleporting photonic qudits using multimode quantum scissors," Scientific Reports $\mathbf{3}$, 3548 (2013).

[7] N. Gisin, G. Ribordy, W. Tittel, and H. Zbinden, "Quantum cryptography," Rev. Mod. Phys. 74, 145 (2002).

[8] K. Bartkiewicz, K. Lemr, A. Cernoch, J. Soubusta, and A. Miranowicz, "Experimental eavesdropping based on optimal quantum cloning," Phys. Rev. Lett. 110, 173601 (2013).

[9] G. C. Levine, "Entanglement entropy in a boundary impurity model," Phys. Rev. Lett. 93, 266402 (2004).

[10] A. Kitaev and J. Preskill, "Topological entanglement entropy," Phys. Rev. Lett. 96, 110404 (2006).

[11] M. Bartkowiak, A. Miranowicz, X. Wang, Y. X. Liu, W. Leoński, and F. Nori, "Sudden vanishing and reappearance of nonclassical effects: General occurrence of 
finite-time decays and periodic vanishings of nonclassicality and entanglement witnesses," Phys. Rev. A 83, 053814 (2011).

[12] S. Ghosh, T. F. Rosenbaum, G. Aeppli, and S. N. Coppersmith, "Entangled quantum state of magnetic dipoles," Nature 425, 48 (2003).

[13] A. Osterloh, L. Amico, G. Falci, and R. Fazio, "Scaling of entanglement close to a quantum phase transition," Nature 416, 608 (2002).

[14] T. J. Osborne and M. A. Nielsen, "Entanglement in a simple quantum phase transition," Phys. Rev. A 66, 032110 (2002).

[15] L. Amico, R. Fazio, A. Osterloh, and V. Vedral., "Entanglement in many-body systems," Rev. Mod. Phys. 80, 517 (2008).

[16] X. Peng, J. Du, and D. Suter, "Quantum phase transition of ground-state entanglement in a heisenberg spin chain simulated in an nmr quantum computer," Phys. Rev. A 71, 012307 (2005).

[17] J. Zhang, X. Peng, N. Rajendran, and D. Suter, "Detection of quantum critical points by a probe qubit," Phys. Rev. Lett. 100, 100501 (2008).

[18] M. Lewenstein, A. Sanpera, and V. Ahufinger, Ultracold atoms in optical lattices: Simulating quantum many-body systems (Oxford University Press, Oxford, UK, 2012).

[19] R. Blatt and C. F. Roos, "Quantum simulations with trapped ions," Nat. Phys. 8, 277 (2012).

[20] I. Bloch, J. Dalibard, and S. Nascimbene, "Quantum simulations with ultracold quantum gases," Nat. Phys. 8, 267 (2012).

[21] D. Loss and D. P. DiVincenzo, "Quantum computation with quantum dots," Phys. Rev. A 57, 120 (1998).

[22] M. Greiner, O. Mandel, T. Esslinger, T. W. Hansch, and I. Bloch, "Quantum phase transition from a superfluid to a mott insulator in a gas of ultracold atoms," Nature 415, 39 (2002).

[23] I. Bloch, J. Dalibard, and W. Zwerger, "Many-body physics with ultracold gases," Rev. Mod. Phys. 80, 885 (2008).

[24] C. Hung, X. Zhang, N. Gemelke, and C. Chin, "Observation of scale invariance and universality in twodimensional bose gases," Nature 470, 236 (2011).

[25] G. M. Nikolopoulos, D. Petrosyan, and P. Lambropoulos, "Coherent electron wavepacket propagation and entanglement in array of coupled quantum dots," Europhys. Lett. 65, 297 (2004).

[26] M. Christandl, N. Datta, A. Ekert, and A. J. Landahl, "Perfect state transfer in quantum spin networks," Phys. Rev. Lett. 92, 187902 (2004).

[27] L.-A. Wu, A. Miranowicz, X. B. Wang, Y. X. Liu, and F. Nori, "Perfect function transfer and interference effects in interacting boson lattices," Phys. Rev. A 80, 012332 (2009).

[28] C. Weitenberg, M. Endres, J. F. Sherson, M. Cheneau, P. Schauß, T. Fukuhara, I. Bloch, and S. Kuhr, "Singlespin addressing in an atomic mott insulator," Nature 471, 319 (2011).

[29] J. Pietraszewicz, T. Sowiński, M. Brewczyk, M. Lewenstein, and M. Gajda, "Spin dynamics of two bosons in an optical lattice site: A role of anharmonicity and anisotropy of the trapping potential," Phys. Rev. A 88, 013608 (2013).

[30] R. P. Feynman, "Simulating physics with computers," Int. J. Theor. Phys. 21, 467 (1982).
[31] P. Hauke, F. M. Cucchietti, L. Tagliacozzo, I. Deutsch, and M. Lewenstein, "Can one trust quantum simulators?" Rep. Prog. Phys. 75, 082401 (2012).

[32] D. Jaksch, C. Bruder, J. I. Cirac, C. W. Gardiner, and P. Zoller, "Cold bosonic atoms in optical lattices," Phys. Rev. Lett. 81, 3108 (1998).

[33] O. E. Alon, A. I. Streltsov, and L. S. Cederbaum, "Zoo of quantum phases and excitations of cold bosonic atoms in optical lattices," Phys. Rev. Lett. 95, 030405 (2005).

[34] V. W. Scarola and S. Das Sarma, "Quantum phases of the extended bose-hubbard hamiltonian: Possibility of a supersolid state of cold atoms in optical lattices," Phys. Rev. Lett. 95, 033003 (2005).

[35] F. Pinheiro, J.-P. Martikainen, and J. Larson, "Confined p-band bose-einstein condensates," Phys. Rev. A 85, 033638 (2012).

[36] T. Sowiński, M. Łącki, O. Dutta, J. Pietraszewicz, P. Sierant, M. Gajda, J. Zakrzewski, and M. Lewenstein, "Tunneling-induced restoration of the degeneracy and the time-reversal symmetry breaking in optical lattices," Phys. Rev. Lett. 111, 215302 (2013).

[37] T. Sowiński, "Creation on demand of higher orbital states in a vibrating optical lattice," Phys. Rev. Lett. 108, 165301 (2012).

[38] A. Auerbach, Interacting Electrons and Quantum Magnetism (Springer-Verlag, Berlin, German, 1994).

[39] J. Simon, W. S. Bakr, R. Ma, M. E. Tai, P. M. Preiss, and M. Greiner, "Quantum simulation of antiferromagnetic spin chains in an optical lattice," Nature 472, 307 (2011).

[40] P. W. Anderson, "New approach to the theory of superexchange interactions," Phys. Rev. 115, 2 (1959).

[41] A. Imambekov, M. Lukin, and E. Demler, "Spinexchange interactions of spin-one bosons in optical lattices: Singlet, nematic, and dimerized phases," Phys. Rev. A 68, 063602 (2003).

[42] S.-K. Yip, "Dimer state of spin-1 bosons in an optical lattice," Phys. Rev. Lett. 90, 250402 (2003).

[43] C. Wu, "Hidden symmetry and quantum phases in spin3/2 cold atomic systems," Mod. Phys. Lett. B 20, 1707 (2006).

[44] K. Eckert, L. Zawitkowski, M. J. Leskinen, A. Sanpera, and M. Lewenstein, "Ultracold atomic bose and fermi spinor gases in optical lattices," New J. Phys. 9, 133 (2007).

[45] M. Hermele, V. Gurarie, and A. M. Rey, "Mott insulators of ultracold fermionic alkaline earth atoms: Underconstrained magnetism and chiral spin liquid," Phys. Rev. Lett. 103, 135301 (2009).

[46] J. J. Garcia-Ripoll, M. A. Martin-Delgado, and J. I. Cirac, "Implementation of spin hamiltonians in optical lattices," Phys. Rev. Lett. 93, 250405 (2004).

[47] A. Drzewiński and J. M. J. van Leeuwen, "Renormalization of the ising model in a transverse field," Phys. Rev. B 49, 403 (1994).

[48] A. Drzewiński and R. Dekeyser, "Renormalization of the anisotropic linear xy model," Phys. Rev. B 51, 15218 (1995).

[49] L. Zhou, H. S. Song, Y. Q. Guo, and C. Li, "Enhanced thermal entanglement in an anisotropic heisenberg xyz chain," Phys Rev. A 68, 024301 (2003).

[50] T. Hirano and Y. Hatsugai, "Entanglement entropy of one-dimensional gapped spin chains," J. Phys. Soc. Jpn. 76, 074603 (2007).

[51] X. Peng, J. Zhang, J. Du, and D. Suter, "Ground-state 
entanglement in a system with many-body interactions," Phys. Rev. A 81, 042327 (2010).

[52] J. L. Guo and H. S. Song, "Entanglement and teleportation through a two-qubit heisenberg xxz model with the dzyaloshinskii-moriya interaction," Eur. Phys. J. D 56, 265 (2010).

[53] K. Rodriguez, A. Argüelles, A. K. Kolezhuk, L. Santos, and T.Vekua, "Field-induced phase transitions of repulsive spin-1 bosons in optical lattices," Phys. Rev. Lett. 106, 105302 (2011).

[54] G. De Chiara, M. Lewenstein, and A. Sanpera, "Bilinearbiquadratic spin-1 chain undergoing quadratic zeeman effect," Phys. Rev. B 84, 054451 (2011).

[55] P. Chen, Z.-L. Xue, I. P. McCulloch, M.-C. Chung, and S.-K. Yip, "Dimerized and trimerized phases for spin-2 bosons in a one-dimensional optical lattice," Phys. Rev. A 85, 011601(R) (2012).

[56] P. Millet, F. Mila, F. C. Zhang, M. Mambrini, A. B. Van Oosten, V. A. Pashchenko, A. Sulpice, and A. Stepanov, "Biquadratic interactions and spin-peierls transition in the spin-1 chain $\mathrm{LiVGe}_{2} \mathrm{O}_{6}$," Phys. Rev. Lett. 83, 4176 (1999).

[57] J. Lou, T. Xiang, and Z. Su, "Thermodynamics of the bilinear-biquadratic spin-one heisenberg chain," Phys. Rev. Lett. 85, 2380 (2000).

[58] R. Bastardis, N. Guihèry, and C. de Graaf, "Microscopic origin of isotropic non-heisenberg behavior in $s=1$ magnetic systems," Phys. Rev. B 76, 132412 (2007).

[59] A. Bencini and F. Totti, "On the importance of the biquadratic terms in exchange coupled systems: A posthf investigation," Inorganica Chimica Acta 361, 4153 (2008).

[60] V. V. Semenaka, O. V. Nesterova, V. N. Kokozay, V. V. Dyakonenko, R. I. Zubatyuk, O. Shishkin, R. Boča, J. Jezierska, and A. Ozarowski, " $\mathrm{Cr}^{I I I}-\mathrm{Cr}^{I I I}$ interactions in two alkoxo-bridged heterometallic $\mathrm{Zn}_{2} \mathrm{Cr}_{2}$ complexes self-assembled from zinc oxide, reineckes salt, and diethanolamine," Inorg. Chem. 49, 5460 (2010).

[61] A. Wagner, C. Bruder, and E. Demler, "Spin-1 atoms in optical superlattices: Single-atom tunneling and entanglement," Phys. Rev. A 84, 063636 (2011).

[62] D. J. Papoular, G. V. Shlyapnikov, and J. Dalibard, "Microwave-induced fano-feshbach resonances," Phys. Rev. A 81, 041603(R) (2010).

[63] Y. X. Liu, A. Miranowicz, Y. B. Gao, J. J. Bajer, C. P. Sun, and F. Nori, "Qubit-induced phonon blockade as a signature of quantum behavior in nanomechanical resonators," Phys. Rev. A 82, 032101 (2010).

[64] T. V. Gevorgyan, A. R. Shahinyan, and G. Y. Kryuchkyan, "Generation of fock states and qubits in periodically pulsed nonlinear oscillators," Phys. Rev. A 85, 053802 (2012).

[65] A. Miranowicz, M. Paprzycka, Y. X. Liu, J. Bajer, and F. Nori, "Two-photon and three-photon blockades in driven nonlinear systems," Phys. Rev. A 87, 023809 (2013).

[66] W. Leoński and R. Tanaś, "Possibility of producing the one-photon state in a kicked cavity with a nonlinear kerr medium," Phys. Rev. A 49, R20 (1994).

[67] A. Miranowicz and W. Leoński, "Dissipation in systems of linear and nonlinear quantum scissors," Journal of Optics B: Quant. Semiclass. Opt. 6, S43 (2004).

[68] A. Kowalewska-Kudłaszyk and W. Leoński, "Squeezed vacuum reservoir effect for entanglement decay in the nonlinear quantum scissor system," J. Phys. B: At. Mol. Opt. Phys. 43, 205503 (2010).

[69] W. Leoński and A. Kowalewska-Kudłaszyk, "Quantum scissors finite-dimensional states engineering," Progress in Optics 56, 131 (2011).

[70] G. J. Milburn, "Coherence and chaos in a quantum optical system," Phys. Rev. A 41, 6567 (1990).

[71] G. J. Milburn and C. A. Holmes, "Quantum coherence and classical chaos in a pulsed parametric oscillator with a kerr nonlinearity," Phys. Rev. A 44, 4704 (1991).

[72] W. Leoński, "Quantum and classical dynamics for a pulsed nonlinear oscillator," Physica A: Statistical Mechanics and its Applications 233, 365 (1996).

[73] A. Kowalewska-Kudłaszyk, J. K. Kalaga, and W. Leoński, "Long-time fidelity and chaos for a kicked nonlinear oscillator system," Phys. Lett. A 373, 1334 (2009).

[74] T. V. Gevorgyan, A. R. Shahinyan, L. Y. Chew, and G. Y. Kryuchkyan, "Bistability and chaos at low levels of quanta," Phys. Rev. E 88, 022910 (2013).

[75] R. Grobe, K. Rzążewski, and J. H. Eberly, "Measure of electron-electron correlations in atomic physics," J. Phys. B 27, L503 (1994).

[76] T. Sowiński, M. Brewczyk, M. Gajda, and K. Rzążewski, "Dynamics and decoherence of two cold bosons in a onedimensional harmonic trap," Phys. Rev. A 82, 053631 (2010).

[77] P. Rungta, W. J. Munro, K. Nemoto, P. Deuar, G. J. Milburn, and C. M. Caves, "Qudit entanglement," in "DIRECTIONS IN QUANTUM OPTICS," , vol. 561 of LECTURE NOTES IN PHYSICS, H. J. Carmichael and R. J. Glauber and M. O. Scully, ed. (2001), vol. 561 of LECTURE NOTES IN PHYSICS, pp. 149-164. TAMUONR Workshop on Quantum Optics, JACKSON, WY, JUL 26-30, 1999.

[78] D. Sych and G. Leuchs, "A complete basis of generalized bell states," New J. Phys. 11, 013006 (2009). 